\title{
Proteomics: The Next Frontier-A Biotech Perspective
}

\author{
M. Walid Qoronfleh* \\ Drug Discovery \& Proteomics, Perbio Science, Bioresearch Division, Pierce Biotechnology, Inc, \\ 3747 N. Meridian Rd, PO Box 117, Rockford, IL 61105, USA
}

Proteomics is a nascent technology with much to develop and much to offer. Both the biotechnology and pharmaceutical arenas will be highly impacted by the new window proteomics is opening. This area of studies will permit us to identify, characterize, and quantify proteins on a massive scale, resulting in a fundamental change in biological and chemical approaches to understanding proteins and even the ability to address new important questions. Advancing the tools of proteomic technology further is of major interest to biotech and pharma communities. Proteomics does require substantial resources and infrastructure investment; therefore, the effort will be greatly enhanced by the coordinated participation of all involved, in both academia and industry. Such resources and coordination are only slowly becoming available on the scale necessary to make a significant impact and the Human Proteome Organization (HUPO) is at the vanguard of these efforts. The focus of this piece is on the promises and the challenges of proteomics in biotechnology, drug discovery, and diagnostics. Additional viewpoints from a business and scientific strategic standpoint on where things are headed in the proteomics marketplace will be discussed.

Now that the human genome has been sequenced, the study of the proteins that carry out the gene's instruction is likely to be the next frontier in biomedical research. Surprisingly, the human genome appears to contain perhaps fewer than 30000 genes, which is far fewer than the original estimates. Enter the world of proteomics. Proteomics includes not only the identification and location of proteins, but also the determination of networks, interactions, activities, and functions. A single gene can encode multiple proteins through a variety of translational and post-translational mechanisms. In addition, the same protein can assume multiple forms. Finally, the same protein may have multiple functions, depending on its cellular environment and associated networks. All of these possibilities result in a proteome (the ensemble of proteins related to a genome) estimated to be at least an order of magnitude more complex than the genome itself.

\section{DRUG DISCOVERY}

Drug discovery is a prolonged process that uses a variety of tools from diverse fields. Proteomics as an interdisciplinary field combines both biology and chemistry and the emerging areas of high-throughput automation and bioinformatics. Promising technologies, as part of the arsenal of proteomic techniques, are advancing the utility of proteomics in drug discovery process. The discipline of proteomics is currently divided into three distinct classes that are indispensable to drug discovery: profiling proteomics, functional proteomics, and structural proteomics. The tools in protein drug discovery range from $2 \mathrm{D} /$ mass spec analyses to multiplex protein profiling to structure-based drug design. Vital to the functional/structural proteomics efforts are high-throughput cloning, protein expression, protein production, and protein purification.

\section{BIOTECHNOLOGY}

Experimental studies related to protein fractionation and separation, qualitative and quantitative protein analyses, identification/characterization of the composition of protein complexes and organelles seem likely to enhance our understanding of dynamic cellular processes, thus, making the proteome a less formidable challenge. Looking ahead we expect protein arrays to play a powerful important role and that novel protein therapeutics will emerge.

\section{Protein production}

The challenge of studying proteins in a global way is driving development of new technologies for systematic and comprehensive analysis of protein structure and 
function. Experimental uses of proteins for structural and functional studies typically require milligram amounts in purified form. Protein expression, production, and purification are fundamental processes in these studies; however, they have typically only been applied on a case-by-case basis to proteins of interest. Strategies are emerging to industrialize these processes and overcome the limitations imposed by conventional methods. Parallel processing, miniaturization approaches, array spotting techniques all will significantly contribute to making proteomics more main stream.

\section{Antibodies}

To define the proteome of an organism, there is a need for robust reproducible methods for the quantitative detection of all the polypeptides in a cell. Because of their high-affinity, specificity, and their ability to bind virtually to any protein, antibodies appear particularly promising as the receptor element in protein-detection arrays. For proteomic-scale analyses and fabrication of high-density arrays, the ability to produce and isolate antibodies en masse to a large number of target molecules is critical. The high-throughput issues of recombinant proteins apply equally well to antibody molecules to generate proteins for microarrays and antibodies for immunolocalization and/or functional analysis utilizing highly sensitive detection protocols.

\section{DIAGNOSTIC}

Biomarkers have been in widespread use for many years, but the field has emerged in its own right over the last decade, propelled by proteomics. It is anticipated that biomarker discovery will shift more to analysis of biofluids and, to a lesser extent, tissue samples from relevant patient populations as an initial approach. Furthermore, biomarker panels will be selected based on their prominence in discriminant analysis combined with their amenability to routine standardized clinical tests. Biomarkers derived from proteomics have the potential to dramatically reduce both the time and the risk involved in target discovery and validation, better understand disease mechanisms (novel therapeutic modalities), diagnose and track diseases and drug actions, and, finally, profile patients for personalized medicine. Looking for biomarkers/targets with the right tools in the right place should greatly improve success rates and shorten the development cycle.

\section{HUPO INITIATIVES}

No discussion is complete without mentioning the efforts of HUPO and the various projects that are underway. HUPO has galvanized the scientific community both nationally and internationally to organize several human proteome projects (plasma, liver, brain, etc), even a pilot program for the mouse and rat liver proteome. Under the patronage of HUPO academic labs, industrial partners and regional HUPO societies soon will report significant advances in protein science.

\section{CONCLUDING REMARKS}

Proteomics is changing the way we look at and deal with proteins (diversity in structure, function, and dynamic range). Various instrumentation advancements and analytical tool improvements are a must to achieve the human proteome map. Many of the methods described in the literature were developed either as a proof-of-concept or as a pilot program in response to the proteomic challenge. These technologies have been proven to be able to deliver, to various degrees of course. The effectiveness of current or future methodologies will be determined by resolving the many problems that will be encountered. The success rate with "difficult" proteins will be the true litmus test for any high-throughput, global analyses systems. It is unlikely that the processes in its current form are going to meet the demand for profiling structural or functional proteomic work. Improvements are needed to gain economies of scale and efficiencies in protein manipulation. Parallel processing and generic methods are required to achieve a systematic and thorough evaluation of protein function. The complexity of this effort is compounded by the large number of gene products comprising the proteome. At the end, subprotein populations (subproteome) will become a commodity, which can be delivered as a product rather than a service. This fundamental change is vital if the promises of the proteome are to be realized.

The immediate impact of proteomics and the fruits of proteomics are likely to be the following:

(1) proteomics will complement existing approaches to drug discovery/diagnostics,

(2) rewards of proteomics in early discovery efforts will be realized 8-10 years of the typical drug development cycle,

(3) proteomic studies benefits will be seen sooner at later-stage uses for drugs on or close to market (biomarkers for efficacy, toxicity, diagnostic, etc).

As final thoughts, it is hard to perceive that a certain technology or one system will dominate the landscape of proteomics. Proteomics will become an integral component of systems biology to facilitate better-informed and earlier R\&D decisions. Perhaps proteomics is like housework, it will never end!

M. Walid Qoronfleh 
M. Walid Qoronfleh earned an MS in Chemistry from Illinois and a $\mathrm{PhD}$ in Microbial Genetics from The University of Louisville Medical School. After postdoctoral work at the University of Georgia in molecular biology, he started his career in the pharmaceutical industry in 1989. Later, he received an MBA with an emphasis on R\&D and technology management from Penn State University. As

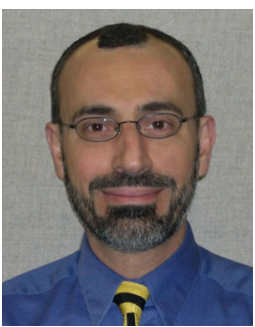
a scientist at SmithKline Beecham, he worked in the areas of heterologous gene expression/protein engineering and studied the molecular aspects of HIV drug resistance. As a Sr. Scientist/Group Leader with Sterling-Winthrop Pharmaceuticals, he played a key role in the development of collagenases as a target for HTS/structure-based drug design studies where the team advanced two drug candidates. As a Scientist Manager in the Structural Biochemistry Program at the NCI his activities focused on functional genomics, antivirals and anticancers where he was involved in target identification/validation and developed assays to several enzymes. He established HT protein expression and protein purification capabilities for structurebased drug design efforts. As a Director of Antiinfective Development at AntexPharma he established the company's HTS capability and was responsible for hit-to-lead and lead optimization efforts. His efforts and the screens identified a broadspectrum and specific agent antibiotics. Currently, he is the Senior Director for Drug Discovery \& Proteomics at Perbio Science Bioresearch Division. He is engaged in various aspects of proteomic research (methods or technology development). These efforts resulted in several commercial products being available in the market place. He has 40 peer-reviewed papers and over 50 published abstracts to his credit. He is a featured speaker at national and international conferences. Recently, M.W. Qoronfleh in collaboration with the Medical College of Wisconsin in Milwaukee, University of WisconsinMadison, and other partners were awarded an NHLBI grant to establish a proteomic center at MCW.

* E-mail: walid.qoronfleh@perbio.com 

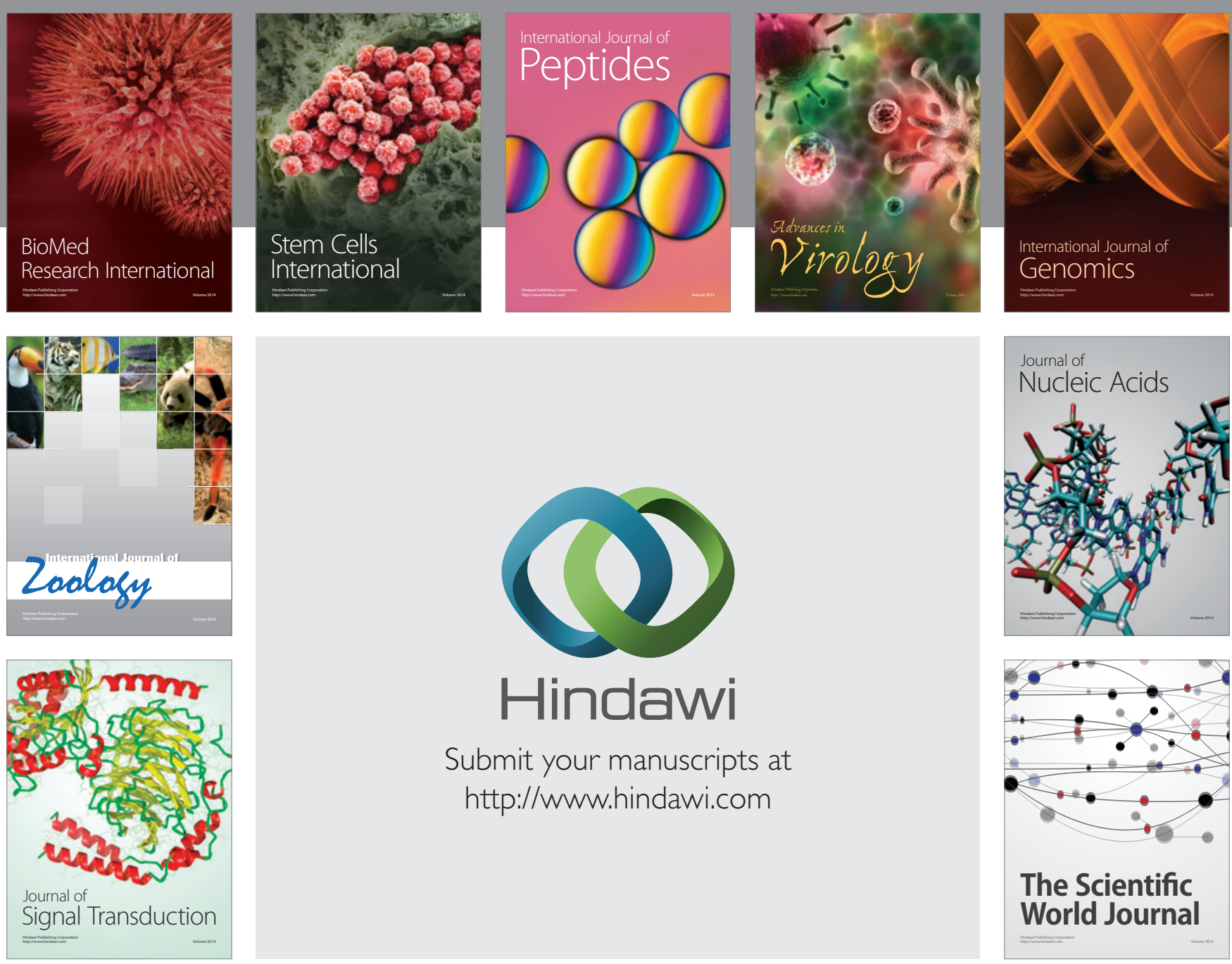

Submit your manuscripts at

http://www.hindawi.com
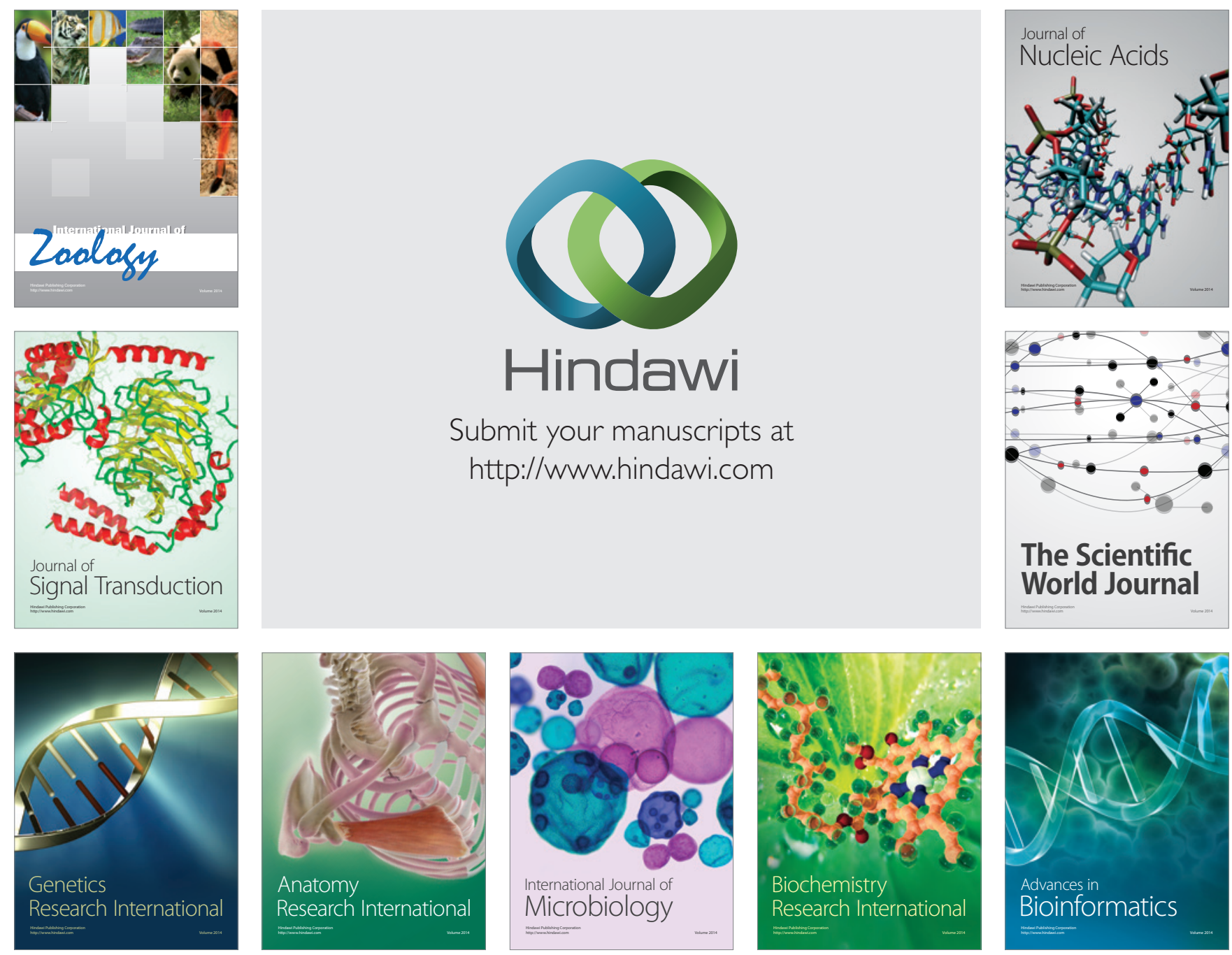

The Scientific World Journal
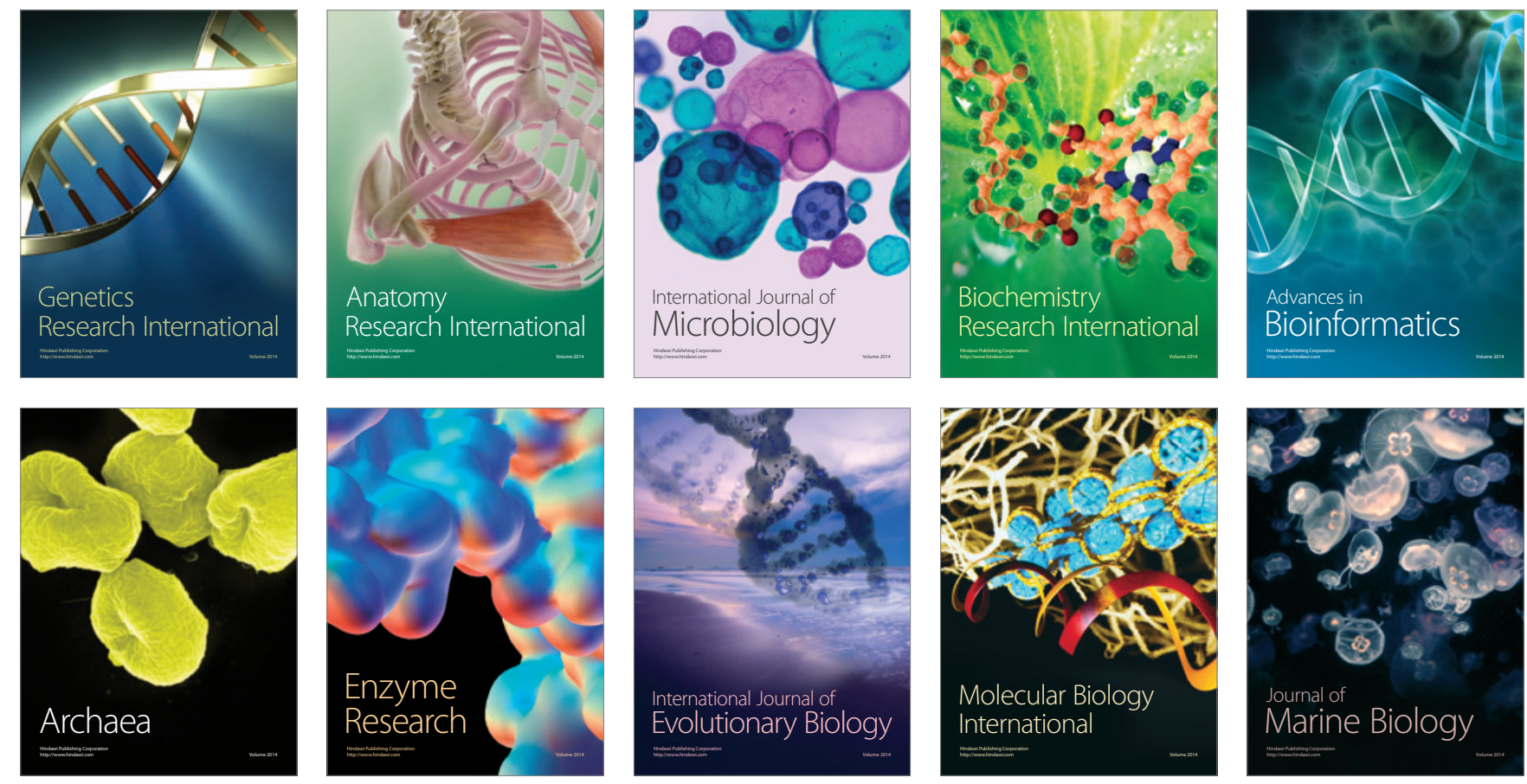